\title{
Study the Entanglement Dynamics of an Anisotropic Two-Qubit Heisenberg XYZ System in a Magnetic Field
}

\author{
A. R. Mohammed, T. M. El-Shahat \\ Mathematics Department, Faculty of Science, Al-Azhar University, Cairo, Egypt \\ Email: a.radwan@azhar.edu.eg, el_shahat@yahoo.com
}

How to cite this paper: Mohammed, A.R. and El-Shahat, T.M. (2017) Study the Entanglement Dynamics of an Anisotropic Two-Qubit Heisenberg XYZ System in a Magnetic Field. Journal of Quantum Information Science, 7, 160-171.

https://doi.org/10.4236/jqis.2017.74013

Received: November 9, 2017

Accepted: December 18, 2017

Published: December 21, 2017

Copyright (c) 2017 by authors and Scientific Research Publishing Inc. This work is licensed under the Creative Commons Attribution International License (CC BY 4.0).

http://creativecommons.org/licenses/by/4.0/

\begin{abstract}
We investigate the entanglement dynamics of an anisotropic two-qubit Heisenberg XYZ system with Dzyaloshinskii-Moriya (DM) interaction in the presence of both inhomogeneity of the external magnetic field $b$ and intrinsic decoherence which has been studied. The behavior of quantum correlation and the degree of entanglement between the two subsystems is quantified by using measurement-induced disturbance (MID), negativity (N) and Quantum Discord (QD), respectively. It is shown that in the presence of an inhomogeneity external magnetic field occur the phenomena of long-lived entanglement. It is found that the initial state is the essential role in the time evolution of the entanglement.
\end{abstract}

\section{Keywords}

Different Dzyaloshinski-Moriya Interactions, Entanglement, Decoherence

\section{Introduction}

Nowadays, correlated systems represent one of the most important partners in the context of quantum communication [1] [2], quantum networks [3] [4] and quantum computers. Probably it is difficult to generate entangled systems with the same dimensions. This task may be difficult if the used devices are imperfect. Moreover, one success to generate maximum entangled states, but keeping them long-lived entangled is a very difficult task and may cost more [5] [6]. These states could be subject to noise channels [7] or dissipative environment [8]. Therefore, in the presence of a different type of noise, it is important to investigate the behavior of entanglement. There are several efforts which have been done to investigate the amount of survival entanglement of different systems 
pass through a different type of noise. Yu and Eberly investigated that the dynamics of entanglement between two qubits system interacting independently with classical or quantum noise displays the phenomena of entanglement decay and entanglement sudden death (ESD) [9] [10]. The dynamics of impurity and entanglement for a bipartite system that passes into Block channels were investigated in [11].

The intrinsic decoherence noise is one of the most important types of noise [12]. For example, M.-L. Hu and H.-L. Lian in [13] have investigated the quantum state transfer and the distribution of entanglement in the model of Milburns intrinsic decoherence. In [14], R. J. Amaro et al. have studied the interaction of a two-level atom and two fields, one of them is classical in the dispersive regime by using a model of intrinsic decoherence. By quantum, the possibility of reducing intrinsic decoherence is a superconducting circuit. Error detection is discussed by Zhong, et al. The effects of an inhomogeneous magnetic field on entanglement and teleportation in a two-qubit XXZ chain with intrinsic decoherence have been investigated [15] [16]. Therefore, we have motivated to investigate the behavior of quantum correlation for the system consists of two different dimensional subsystems in the presences of intrinsic decoherence. We need to investigate the effect of the dimensions of the system that passes through this type of noise on the degree of correlations between its subsystems. They use measurement-induced disturbance (MID), which does not include improvement methodology, to portray correlations as classical or quantum [17].

In this paper, we will investigate the quantum correlations base on MID in our model. As we probably are aware, the quantum entanglement of dense issue frameworks is a vital developing field as previously. Individuals have made a few examinations of quantum entanglement of thermal equilibrium states of spin chains subject to an external magnetic field at finite temperature [18] [19]. Likewise, the quantum correlations of two qubits with Dzyaloshinskii Moriya (DM) interaction, which can impact the phase transition, additionally have pulled in much consideration [20]. In this paper, in the examination with the warm quantum discord of two qubits [21] [22], we not just extend the examination on warm quantum correlation to blended turn $(1 / 2,1)$ XXZ display, yet in addition consider the impacts of DM interaction and outside magnetic field on a thermal quantum correlation measured by MID [23].

The rest of this paper is organized as follows. In Section 2, we introduce the Hamiltonian of the Heisenberg model with different DM interaction and present the exact solution of the model. In Section 3, is devoted to investigating the dynamics of entanglement and quantum correlation by means of negativity, measurement-induced disturbance [24] and Quantum Discord, respectively, Finally, we summarize our results in Section 4.

\section{Model and Solution}

The Hamiltonian $H$ for a two-qubit anisotropic Heisenberg model with z-component interaction parameter $D_{z}$ is 


$$
\begin{aligned}
H= & J(1+\gamma) \sigma_{1}^{x} \sigma_{2}^{x}+J(1-\gamma) \sigma_{1}^{y} \sigma_{2}^{y}+J_{z} \sigma_{1}^{z} \sigma_{2}^{z}+D_{z}\left(\sigma_{1}^{x} \sigma_{2}^{y}-\sigma_{1}^{y} \sigma_{2}^{x}\right) \\
& +(B+b) \sigma_{1}^{z}+(B-b) \sigma_{2}^{z}
\end{aligned}
$$

where $J$ and $J_{z}$ are the real coupling coefficients, $\gamma$ is the anisotropic parameter. $D_{z}$ is the z-component DM interaction parameter, and $\sigma^{i}(i=x, y, z)$ are Pauli matrices. $B$ is the homogeneous part of the magnetic field and $b$ describes the inhomogenity. The external magnetic fields and Dzyaloshinskii Moriya interaction are assumed to be along the z-direction. All the parameters are dimensionless. We get on the eigenvalues of the Hamiltonian $H$ are given by

$$
\begin{aligned}
& E_{1}=J_{z}+2 \sqrt{B^{2}+J^{2} \gamma^{2}} \\
& E_{2}=J_{z}-2 \sqrt{B^{2}+J^{2} \gamma^{2}} \\
& E_{3}=-J_{z}+2 \sqrt{b^{2}+D_{z}^{2}+J^{2}} \\
& E_{4}=-J_{z}-2 \sqrt{b^{2}+D_{z}^{2}+J^{2}}
\end{aligned}
$$

We get on the eigenvectors of the Hamiltonian $H$ are given by

$$
\begin{aligned}
& \left|\psi_{1}\right\rangle=\frac{1}{\sqrt{1+\mu^{2}}}(\mu|11\rangle+|00\rangle) \\
& \left|\psi_{2}\right\rangle=\frac{1}{\sqrt{1+v^{2}}}(v|11\rangle+|00\rangle) \\
& \left|\psi_{3}\right\rangle=\frac{1}{\sqrt{1+X}}(N|10\rangle+|01\rangle) \\
& \left|\psi_{4}\right\rangle=\frac{1}{\sqrt{1+Y}}(M|10\rangle+|01\rangle)
\end{aligned}
$$

where,

$$
\begin{gathered}
\mu=\frac{B+\sqrt{B^{2}+J^{2} \gamma^{2}}}{J \gamma}, \quad \theta=b+\sqrt{b^{2}+D_{z}^{2}+J^{2}} \\
v=\frac{B+\sqrt{B^{2}+J^{2} \gamma^{2}}}{J \gamma}, \quad \phi=-b+\sqrt{b^{2}+D_{z}^{2}+J^{2}} \\
X=\frac{\theta^{2}}{D z^{2}+J^{2}}, \quad Y=\frac{\phi^{2}}{D z^{2}+J^{2}} \\
N=\frac{\theta\left(-I D_{z}+J\right)}{D_{z}^{2}+J^{2}}, \quad M=\frac{\phi\left(I D_{z}-J\right)}{D_{z}^{2}+J^{2}}
\end{gathered}
$$

The master equation describing the intrinsic decoherence under the Markovian approximations is given by

$$
\frac{\mathrm{d} \rho(t)}{\mathrm{d} t}=-i[H, \rho(t)]-\frac{\Gamma}{2}[H,[H, \rho(t)]]
$$

where $\Gamma$ is the intrinsic decoherence rate. The formal solution of the above master equation can be expressed as 


$$
\rho(t)=\sum_{k=0}^{\infty} \frac{(\Gamma t)^{k}}{k !} M^{k} \rho(0) M^{+k}
$$

where $\rho(0)$ is the density operator of the initial system and $M^{k}$ is defined by

$$
M^{k}=H^{k} \mathrm{e}^{-i H t} \mathrm{e}^{-\frac{\Gamma t}{2} H^{2}}
$$

According to Equation (9) it is easy to show that, under intrinsic decoherence, the dynamics of the density operator $\rho(t)$ for the above-mentioned system which is initially in the state $\rho(0)$ is given by

$$
\begin{aligned}
\rho(t)= & \sum_{m n} \exp \left[-\frac{\Gamma t}{2}\left(E_{m}-E_{n}\right)^{2}-i\left(E_{m}-E_{n}\right) t\right] \\
& \times\left\langle\psi_{m}|\rho(0)| \psi_{n}\right\rangle\left|\psi_{m}\right\rangle\left\langle\psi_{n}\right|
\end{aligned}
$$

where $E_{m}, E_{n},\left|\psi_{m}\right\rangle,\left|\psi_{n}\right\rangle$ are the eigenvalues and the corresponding eigenvectors of $H$. In the standard basis $\{|00\rangle,|01\rangle,|10\rangle,|11\rangle\}$ the time evolution of the density operator of the system will be obtained for two different initial states as:

The two qubits are initially in an entangled state $\rho(0)=|\phi\rangle\langle\phi|$, $|\phi\rangle=\cos (\alpha)|01\rangle+\sin (\alpha)|10\rangle$, we get

$$
\begin{aligned}
& \rho(t)=\left(\begin{array}{cccc}
0 & 0 & 0 & 0 \\
0 & \rho_{22} & \rho_{23} & 0 \\
0 & \rho_{32} & \rho_{33} & 0 \\
0 & 0 & 0 & 0
\end{array}\right) \\
& \rho_{22}=\frac{1}{4 y^{2}}[((b+y)(y-b \cos (2 \alpha)+J \sin (2 \alpha))) \\
& -\left(\mathrm{e}^{-4 i y t-8 y^{2} t \Gamma}\left(\left(D_{z}^{2}+J^{2}\right) \cos (2 \alpha)+\left(b J+i D_{z} y\right) \sin (2 \alpha)\right)\right) \\
& -\left(\mathrm{e}^{4 i y t-8 y^{2} t \Gamma}\left(\left(D_{z}^{2}+J^{2}\right) \cos (2 \alpha)+\left(b J-i D_{z} y\right) \sin (2 \alpha)\right)\right) \\
& +((-b+y)(y+b \cos (2 \alpha)-J \sin (2 \alpha)))] \\
& \rho_{23}=\frac{1}{4 y^{2}}\left[\left(\left(-i D_{z}+J\right)(y-b \cos (2 \alpha)+J \sin (2 \alpha))\right)\right. \\
& \left.-\left(\left(-i D_{z}+J\right)(y+b \cos (2 \alpha)-J \sin (2 \alpha))\right)\right]+\frac{1}{4\left(i D_{z}+J\right)\left(y^{2}\right)} \\
& \times\left(\left(\mathrm{e}^{-4 i y t-8 y^{2} t \Gamma}(b+y)\left(\left(D_{z}^{2}+J^{2}\right) \cos (2 \alpha)+\left(b J+i D_{z} y\right) \sin (2 \alpha)\right)\right)\right. \\
& \left.+\left(\mathrm{e}^{4 i y t-8 y^{2} t \Gamma}(b-y)\left(\left(D_{z}^{2}+J^{2}\right) \cos (2 \alpha)+\left(b J-i D_{z} y\right) \sin (2 \alpha)\right)\right)\right) \\
& \rho_{32}=\frac{1}{4 y^{2}}\left[\left(\left(i D_{z}+J\right)\left(\sqrt{b^{2}+D_{z}^{2}+J^{2}}-b \cos (2 \alpha)+J \sin (2 \alpha)\right)\right)\right. \\
& \left.-\left(\left(i D_{z}+J\right)(y+b \cos (2 \alpha)-J \sin (2 \alpha))\right)\right]+\frac{1}{4\left(i D_{z}+J\right)\left(y^{2}\right)} \\
& \times\left[\left(\mathrm{e}^{-4 i y t-8 y^{2}+\Gamma}(b-y)\left(\left(D_{z}^{2}+J^{2}\right) \cos (2 \alpha)+\left(b J+i D_{z} y\right) \sin (2 \alpha)\right)\right)\right. \\
& \left.+\left(\mathrm{e}^{4 i y t-8 y^{2} t \Gamma}(b+y)\left(\left(D_{z}^{2}+J^{2}\right) \cos (2 \alpha)+\left(b J-i D_{z} y\right) \sin (2 \alpha)\right)\right)\right]
\end{aligned}
$$




$$
\begin{aligned}
\rho_{33}= & \frac{1}{4 y^{2}(b+y)}\left[\left(D_{z}^{2}+J^{2}\right)(y-b \cos (2 \alpha)+J \sin (2 \alpha))\right] \\
& +\frac{1}{4 y^{2}(-b+y)}\left[\left(D_{z}^{2}+J^{2}\right)(y+b \cos (2 \alpha)-J \sin (2 \alpha))\right] \\
& +\frac{1}{4 y^{2}}\left[\left(\mathrm{e}^{-4 i y t-8 y^{2} t \Gamma}\left(\left(D_{z}^{2}+J^{2}\right) \cos (2 \alpha)+\left(b J+i D_{z} y\right) \sin (2 \alpha)\right)\right)\right. \\
& \left.+\left(\mathrm{e}^{4 i y t-8 y^{2} t \Gamma}\left(\left(D_{z}^{2}+J^{2}\right) \cos (2 \alpha)+\left(b J-i D_{z} y\right) \sin (2 \alpha)\right)\right)\right]
\end{aligned}
$$

\section{Entanglement Evolutions}

\subsection{Negativity}

There is some systems could be entangled but have a zero negativity and non-zero values of quantum correlations. This means that there are some quantum correlation cannot be predicted by using the negativity as a measure of entanglement. However, by using the measurement-induced disturbance as a measure of quantum correlation, one can quantify the unpredicted quantum correlation [24] [25]. Therefore, we are studying the effect of a type of noise called intrinsic decoherence on the negativity and the measurement-induced disturbance in this section, we investigate the behavior of entanglement in the presences of intrinsic decoherence, by means of negativity $\mathrm{N}$. This measure states that if $\left\{\lambda_{\mu}\right\}$ represents the eigenvalues of $\rho_{a b}^{T_{2}}$, then the negativity is given by, $N=\sum_{i=1}^{4}\left|\lambda_{i}\right|-1$ where $T_{2}$ refers to the partial transposition for the second subsystem. By using this definition for our system, the negativity can be calculated explicitly as, $N=\max \left[0,-2 \min \left[\lambda_{i}\right]\right]$. Thus in this letter, we use negativity as our measure of entanglement. The values of $\mathrm{N}$ range from zero to one: For a maximally-entangled when $N=1$, while for a unentangled state $N=0$.

\subsection{Quantum Correlation via Measurement-Induced Disturbance}

By using $\Pi_{k}=\Pi_{i}^{a} \otimes \Pi_{j}^{b}$ and $\Pi_{i}^{a}, \Pi_{j}^{b}$ are complete projective measurements consisting of one-dimensional orthogonal projections for parties a and $\mathrm{b}$, we can apply local measurement $\left\{\Pi_{k}\right\}\left(\Pi_{k} \Pi_{k^{\prime}}=\delta_{k k^{\prime}} \Pi_{k}\right)$ and $\sum_{k} \Pi_{k}=1$, to any bipartite state $\rho$ (of course, including thermal state). After the measurement, we get the state $\Pi(\rho)=\sum_{i j}\left(\Pi_{i}^{a} \otimes \Pi_{j}^{b}\right) \rho\left(\Pi_{i}^{a} \otimes \Pi_{j}^{b}\right)$ which is a classical state. If the measurement $\Pi$ is induced by the spectral resolutions of the reduced states $\rho^{a}=\sum_{i} p_{i}^{a} \Pi_{i}^{a}$ and $\rho^{b}=\sum_{i} p_{i}^{b} \Pi_{i}^{b}$, the measurement leaves the marginal information invariant and is in a certain sense the least disturbing. In fact, $\Pi(\rho)$ is a classical state that is closest to the original state $\rho$ since this kind of measurement can leave the reduced states invariant. One can use any reasonable distance between $\rho$ and $\Pi(\rho)$ to measure the quantum correlation in $\rho$. In this article, we will use Luos method i.e., quantum mutual information difference between $\rho$ and $\Pi(\rho)$, to measure quantum correlation in $\rho$. The total correlation in a bipartite state $\rho$ can be well quantified by the quantum mutual information $I(\rho)=S\left(\rho^{a}\right)+S\left(\rho^{b}\right)-S(\rho)$, and $I(\Pi(\rho))$, quantifies the clas- 
sical correlations in $\rho$ since $\Pi(\rho)$ is a classical state. Here $S(\rho)=-\sum_{i} \lambda_{i} \log _{2} \lambda_{i}$ denotes the von Neumann entropy. So the quantum correlation can be quantified by the measurement induced disturbance $Q(\rho)=$ $I(\rho)-I(\Pi(\rho))$. where

$$
\Pi(\rho)=\left(\begin{array}{cccc}
\rho_{11} & 0 & 0 & 0 \\
0 & \rho_{22} & 0 & 0 \\
0 & 0 & \rho_{33} & 0 \\
0 & 0 & 0 & \rho_{44}
\end{array}\right)
$$

\subsection{Quantum Discord}

Quantum discord is based on the difference between the quantum mutual information and the classical correlation. For a two-qubit quantum system, the total correlation is measured by their quantum mutual information $L\left(\rho_{a b}\right)=S\left(\rho_{a}\right)$ $+S\left(\rho_{b}\right)-S\left(\rho_{a b}\right)$, where $\rho_{a(b)}$ and $\rho_{a b}$ denote the reduced density matrix of $a(b)$ and the density of the bipartite system respectively, and $S(\rho)=$ $-\operatorname{tr}\left(\rho \log _{2} \rho\right)$ is the von Neumann entropy. Quantum discord, which quantifies the quantumness of correlation between $\mathrm{A}$ and $\mathrm{B}$, is then defined as the difference between the total correlation and classical correlation. For the $\mathrm{X}$ state described by the density matrix quantum discord (QD) is given as

$$
Q D=\min \left[D_{1}, D_{2}\right]
$$

with

$$
Q D_{j}=\Gamma\left(\rho_{11}+\rho_{33}\right)+\sum_{i=1}^{4} \lambda_{i} \log _{2} \lambda_{i}+R_{j}
$$

and

$$
\begin{gathered}
R_{1}=-\Gamma\left(\rho_{11}+\rho_{33}\right)-\sum_{i=1}^{4} \rho_{i i} \log _{2} \rho_{i i}, \\
R_{2}=\Gamma(p) \\
p=\frac{1+\sqrt{\left[1-2\left(\rho_{33}+\rho_{44}\right)\right]^{2}+4\left(\left|\rho_{14}\right|+\left|\rho_{23}\right|\right)^{2}}}{2}
\end{gathered}
$$

where $\lambda_{i}$ being the four eigenvalues of the density matrix $\rho$ and $\Gamma(x)=$ $-x \log _{2} x-(1-x) \log _{2}(1-x)$. As the density matrix of our system in Equation (9) is $X$ states, quantum discord can be evaluated by substituting from Equation (9) into Equation (11) after straightforward calculation, quantum discord reads

$$
Q D=\min \left[D_{1}, D_{2}\right]
$$

with

$$
\begin{aligned}
D_{1}= & \left(1 / \log _{2}[4]\right)\left(-\left(\rho_{22}+\rho_{33}\right) \log _{2}[4]-2 \rho_{22} \log _{2}\left[\rho_{22}\right]-2 \rho_{33} \log _{2}\left[\rho_{33}\right]\right. \\
& +\left(\rho_{22}-\sqrt{4 \rho_{23} \rho_{32}+\left(\rho_{22}-\rho_{33}\right)^{2}}+\rho_{33}\right)
\end{aligned}
$$




$$
\begin{aligned}
& \times \log _{2}\left[\rho_{22}-\sqrt{4 \rho_{23} \rho_{32}+\left(\rho_{22}-\rho_{33}\right)^{2}}+\rho_{33}\right] \\
& +\left(\rho_{22}+\sqrt{4 \rho_{23} \rho_{32}+\left(\rho_{22}-\rho_{33}\right)^{2}}+\rho_{33}\right) \\
& \left.\times \log _{2}\left[\rho_{22}+\sqrt{4 \rho_{23} \rho_{32}+\left(\rho_{22}-\rho_{33}\right)^{2}}+\rho_{33}\right]\right) \\
D_{2}= & \frac{1}{\log _{2}[4]}\left(4 p \tanh ^{-1}[1-2 p]+4 \rho_{33} \tanh ^{-1}\left[1-2 \rho_{33}\right]\right. \\
& -\left(\rho_{22}+\rho_{33}\right) \log _{2}[4]-2 \log _{2}[1-p]-2 \log _{2}\left[1-\rho_{33}\right] \\
+ & \left(\rho_{22}-\sqrt{4 \rho_{23} \rho_{32}+\left(\rho_{22}-\rho_{33}\right)^{2}}+\rho_{33}\right) \\
& \times \log \left[\rho_{22}-\sqrt{4 \rho_{23} \rho_{32}+\left(\rho_{22}-\rho_{33}\right)^{2}}+\rho_{33}\right] \\
& +\left(\rho_{22}+\sqrt{4 \rho_{23} \rho_{32}+\left(\rho_{22}-\rho_{33}\right)^{2}}+\rho_{33}\right) \\
& \left.\times \log \left[\rho_{22}+\sqrt{4 \rho_{23} \rho_{32}+\left(\rho_{22}-\rho_{33}\right)^{2}}+\rho_{33}\right]\right)
\end{aligned}
$$

In Figure 1 we plot the negativity $\mathrm{N}$ as a function of time $\mathrm{t}$ with different values DM interaction in the absence of a magnetic field. We see that negativity the movement starts from a point that varies according to different the initial states. We note that negativity rising to the highest value approaching one. We find that it oscillates and decay over time and with increased the DM interaction, it is seen that increased amplitude while reducing frequencies, the negativity $\mathrm{N}$ oscillates for the longest time before it reaches steady-going values. On the other side in the presence of an inhomogeneous external magnetic field, we find that the negativity $\mathrm{N}$, the oscillation period decay and decay faster before reaching a stable values in Figure 2. In Figure 3 we plot the Measurement-induced disturbance (MID) as a function of time $t$ with different values DM interaction in the absence of a magnetic field. We see that MID at the beginning of the track Moves from a point that varies according to Different the initial states. We note that negativity rising to the highest value at 0.62 . We find that it oscillates and decay over time and with increased the DM interaction, it is seen that increased amplitude while reducing frequencies, MID oscillates for the longest time before it reaches steady-going values. On the other side in the presence of an inhomogeneous external magnetic field, we find that MID, the oscillation period decay and decay faster before reaching a stable values not equal to zero in Figure 4. In Figure 5 we plot quantum discord (QD) as a function of time $t$ with different values DM interaction in the absence of a magnetic field. We see that QD At the beginning of the track moves from a point that varies according to different the initial states. We note that negativity rising to the highest value approaching one. We find that it oscillates and decay over time and with increased the DM interaction, it is seen that amplitude is greater with reducing frequencies; QD oscillates for the longest time before it reaches steady-going values. On the other side in the presence of inhomogeneous external magnetic field, we find that MID, the oscillation period decay 

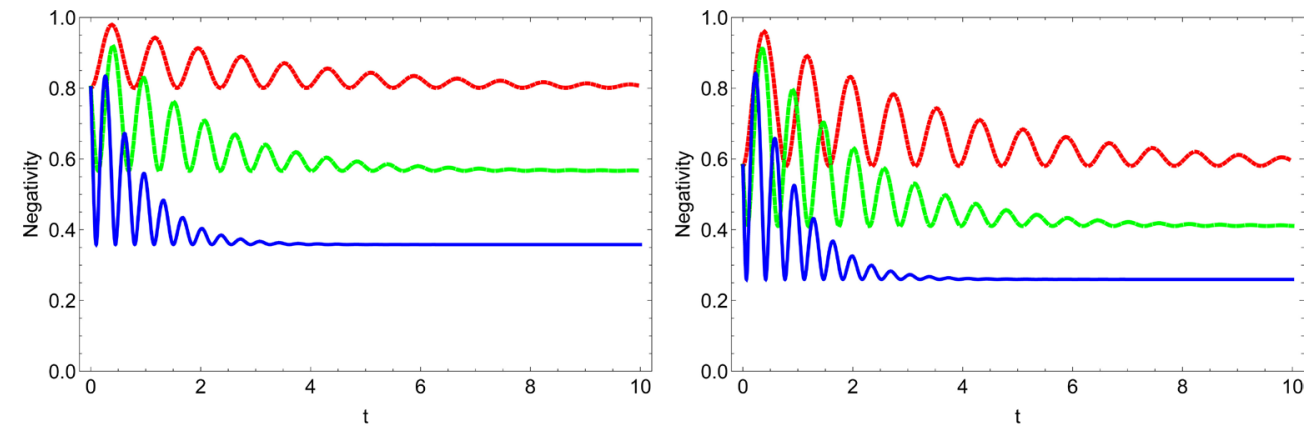

(a) $\alpha=\frac{\pi}{2}$
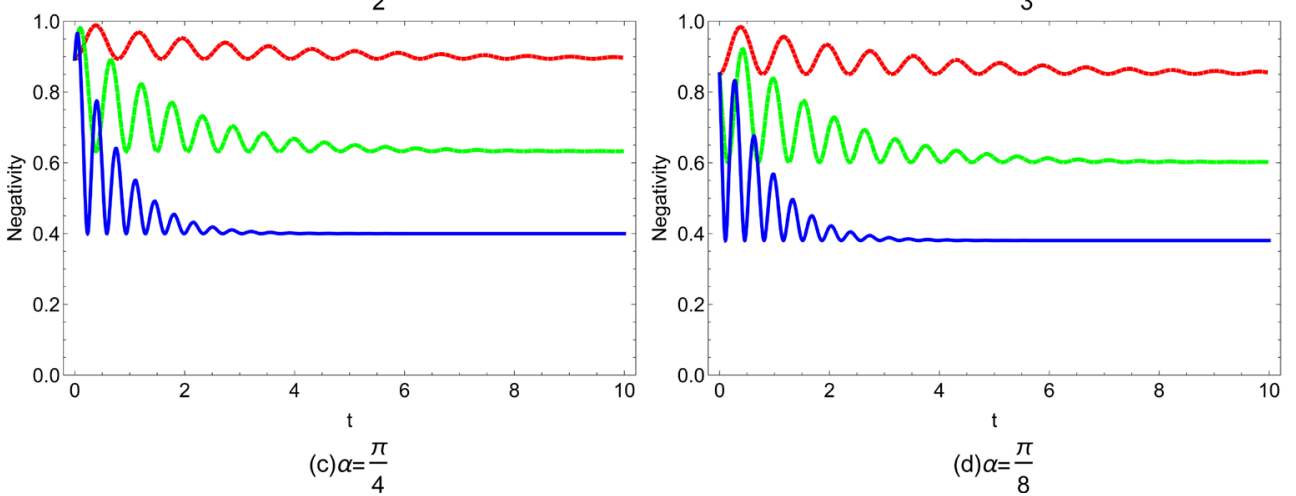

(d) $\alpha=\frac{\pi}{8}$

Figure 1 . The negativity $\mathrm{N}$ as a function of time t. The dotted, dashed and solid curves are evaluated for $D=0,1,2$, respectively in the initial states $\alpha=\frac{\pi}{2}, \frac{\pi}{3}, \frac{\pi}{4}, \frac{\pi}{8}$, respectively $(\gamma=2, \quad J=1, J z=1, \Gamma=0.02, \quad b=0)$.
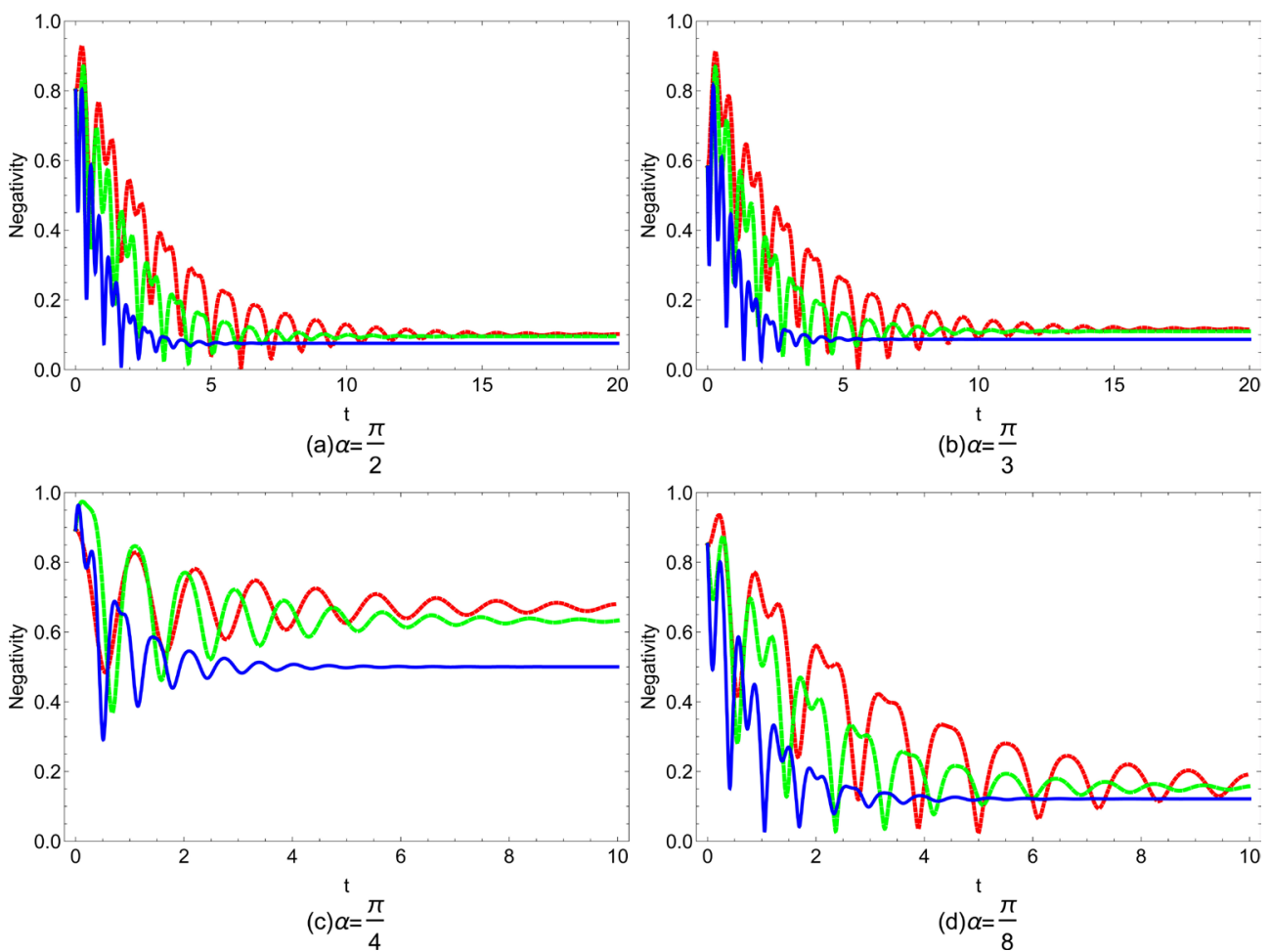

Figure 2. The negativity $\mathrm{N}$ as a function of time t. The dotted, dashed and solid curves are evaluated for $D=0,1,2$, respectively in the initial states $\alpha=\frac{\pi}{2}, \frac{\pi}{3}, \frac{\pi}{4}, \frac{\pi}{8}$, respectively $(\gamma=2, J=1, J z=1, \Gamma=0.02, \quad b=1)$. 

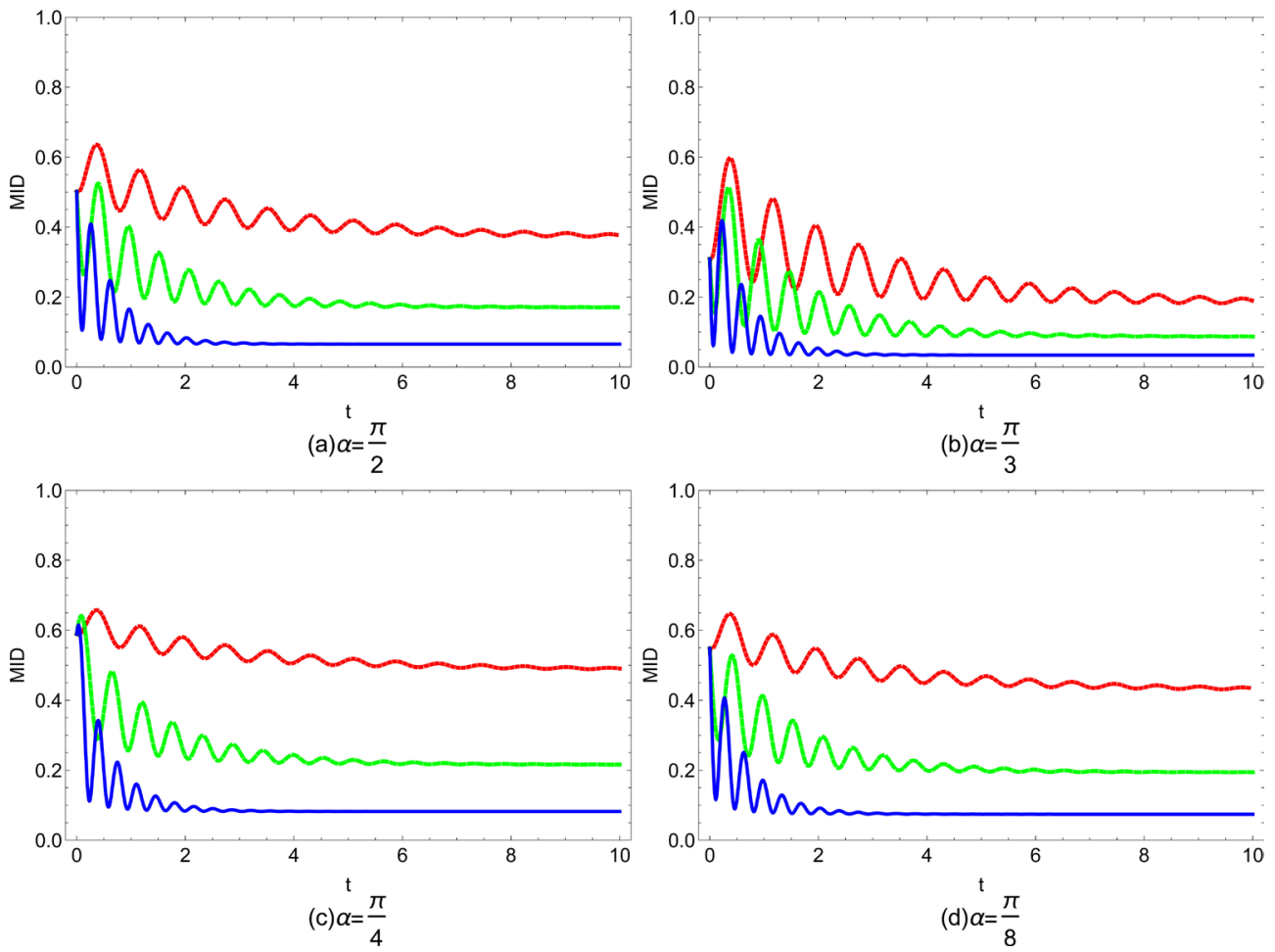

Figure 3. The behavior of measurement-induced disturbance (MID) as a function of time $t$. The dotted, dashed and solid curves are evaluated for $D=0,1,2$, respectively in the initial states $\alpha=\frac{\pi}{2}, \frac{\pi}{3}, \frac{\pi}{4}, \frac{\pi}{8}$, respectively $(\gamma=2, J=1, J z=1, \Gamma=0.02, b=0)$.
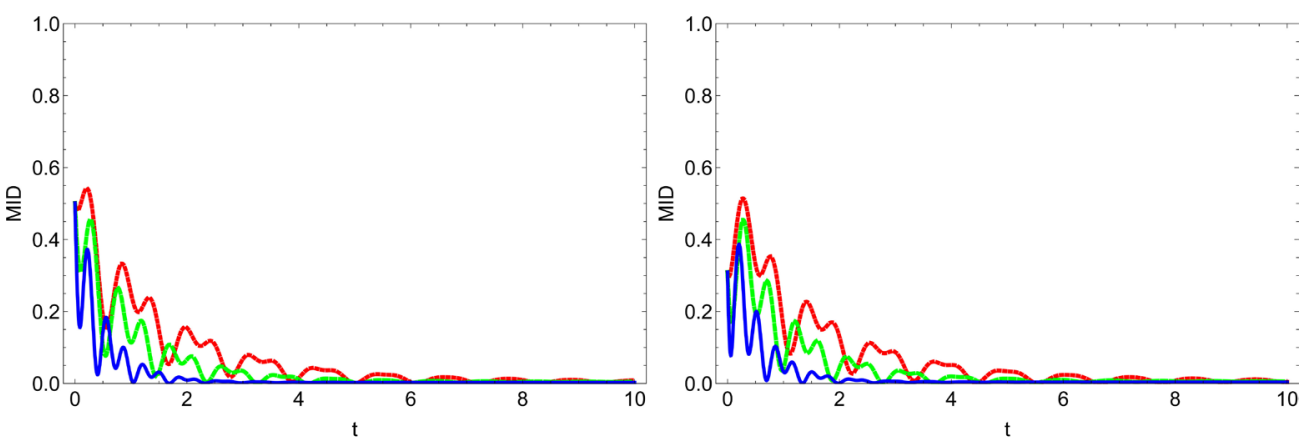

(a) $\alpha=\frac{\pi}{2}$
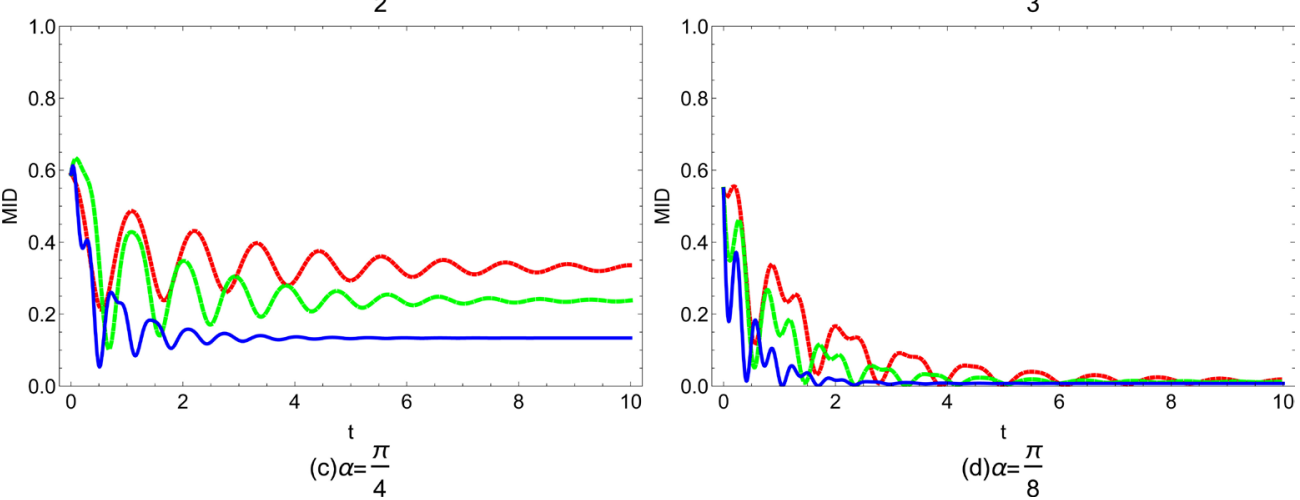

Figure 4. The behavior of measurement-induced disturbance (MID) as a function of time $t$. The dotted, dashed and solid curves are evaluated for $D=0,1,2$, respectively in the initial states $\alpha=\frac{\pi}{2}, \frac{\pi}{3}, \frac{\pi}{4}, \frac{\pi}{8}$, respectively $(\gamma=2, J=1, J z=1, \Gamma=0.02, b=1)$. 

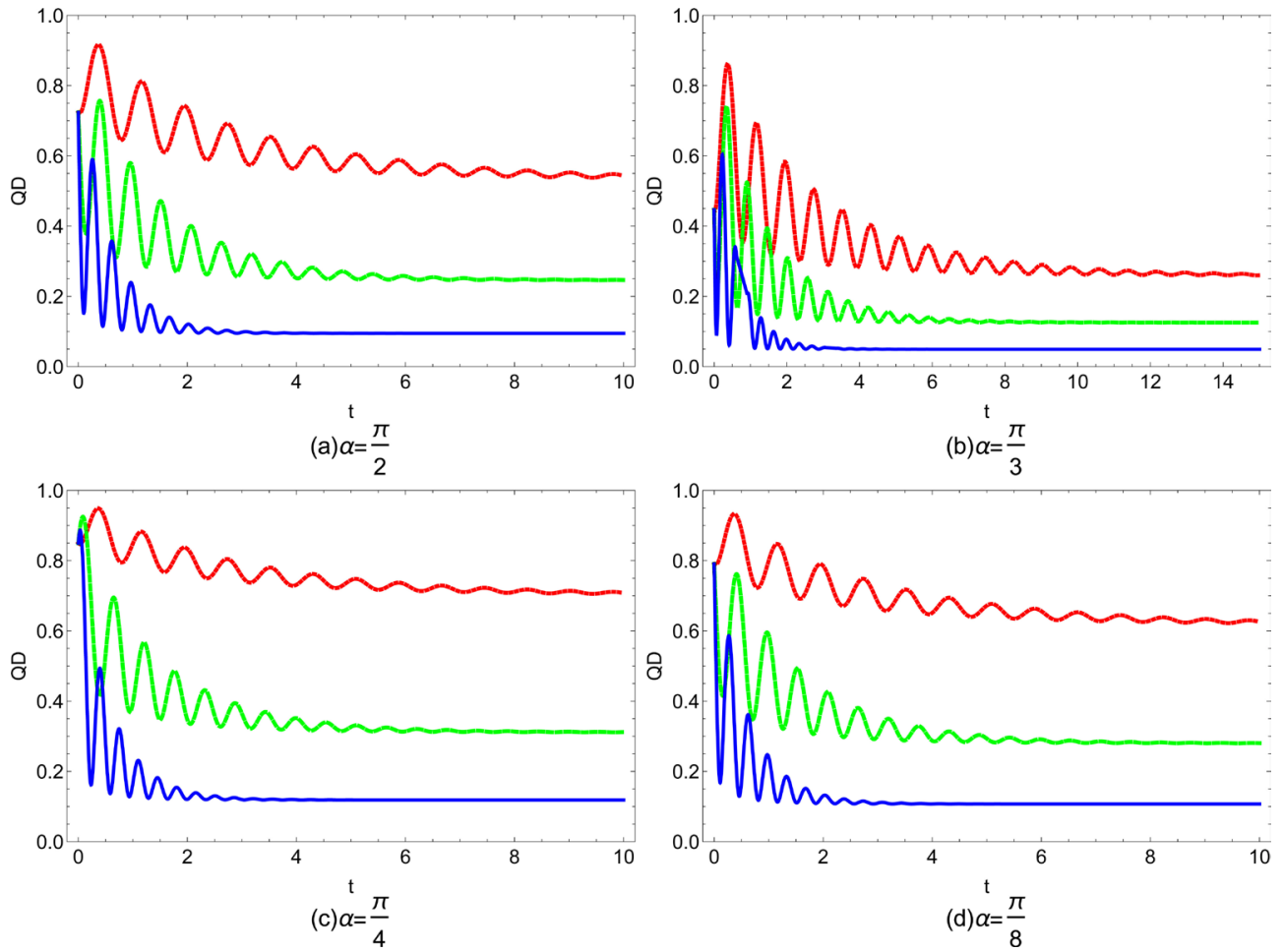

Figure 5. The behavior of Quantum Discord (QD) as a function of time t. The dotted, dashed and solid curves are evaluated for $D=0,1,2$, respectively in the initial states $\alpha=\frac{\pi}{2}, \frac{\pi}{3}, \frac{\pi}{4}, \frac{\pi}{8}$, respectively $(\gamma=2, J=1, J z=1, \Gamma=0.02, \quad b=0)$.
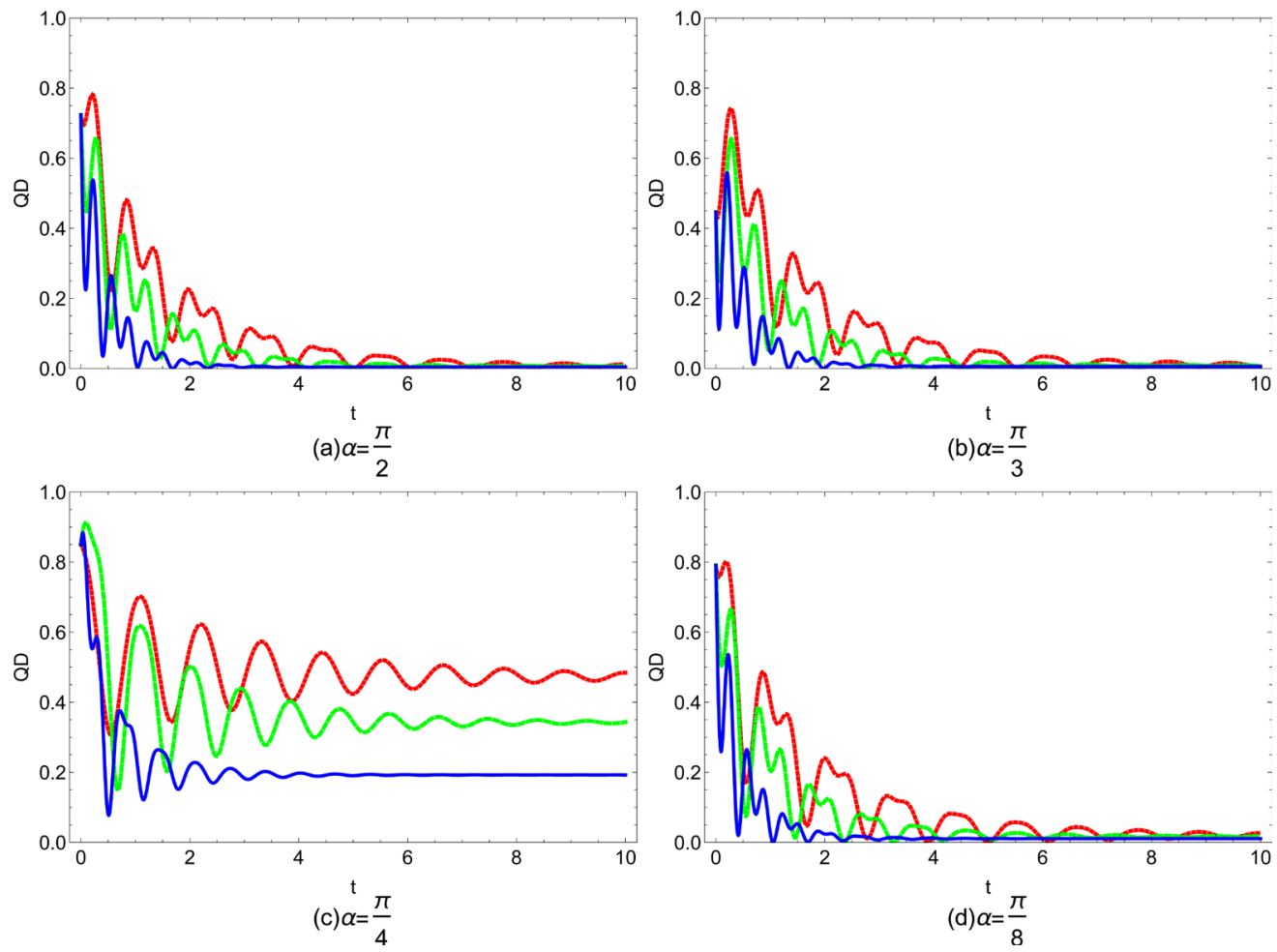

Figure 6. The behavior of Quantum Discord (QD) as a function of time t. The dotted, dashed and solid curves are evaluated for $D=0,1,2$, respectively in the initial states $\alpha=\frac{\pi}{2}, \frac{\pi}{3}, \frac{\pi}{4}, \frac{\pi}{8}$, respectively $(\gamma=2, J=1, J z=1, \Gamma=0.02, \quad b=0)$. 
and decay faster before arrive at a steady-going non-zero value for the long-time case for different values of the DM interaction in Figure 6, which means that the inhomogeneous external magnetic field is a positive component to the entanglement when the partial anisotropic parameter of the system is at a fixed non-zero value. They obtained the similar result that a proper external magnetic field can protect the entanglement from the destructive effect of the intrinsic decoherence.

\section{Conclusion}

In the presence of both external magnetic field and intrinsic decoherence, we have treated the entanglement dynamics of an anisotropic two-qubit Heisenberg XYZ system with Dzyaloshinskii-Moriya interaction which has been studied. We found that the initial state of the system plays an important role in the time evolution of the entanglement. The magnetic field has an effective role in maintaining the intertwining for a long time and non-analysis. The negativity, MID, and QD for different DM interaction will arrive at a steady-going non-zero value for the long-time case, which means that the external magnetic field $b$ is a positive component to the entanglement when the partial anisotropic parameter of the system is at a fixed non-zero value.

\section{References}

[1] Barnett, S. (2009) Quantum Information. Oxford University Press, Oxford, 72.

[2] Sagawa, T. (2012) Introduction. In: Thermodynamics of Information Processing in Small Systems, Springer, Tokyo, 1-7. https://doi.org/10.1007/978-4-431-54168-4_1

[3] Giraud, O., Georgeot, B. and Shepelyansky, D.L. (2005) Quantum Computing of Delocalization in Small-World Networks. Physical Review E, 72, Article ID: 036203. https://doi.org/10.1103/PhysRevE.72.036203

[4] Metwally, N. (2011) Entangled Network and Quantum Communication. Physics Letters A, 375, 4268-4273. https://doi.org/10.1016/j.physleta.2011.10.026

[5] Metwally, N. (2010) Information Loss in Local Dissipation Environments. International Journal of Theoretical Physics, 49, 1571-1579.

https://doi.org/10.1007/s10773-010-0339-9

[6] Bennett, C.H., DiVincenzo, D.P., Smolin, J.A. and Wootters, W.K. (1996) Mixed-State Entanglement and Quantum Error Correction. Physical Review A, 54, 3824-3851. https://doi.org/10.1103/PhysRevA.54.3824

[7] Huang, P., Zhu, J., He, G. and Zeng, G. (2012) Study on the Security of Discrete-Variable Quantum Key Distribution over Non-Markovian Channels. Journal of Physics B, 45, Article ID: 135501. https://doi.org/10.1088/0953-4075/45/13/135501

[8] Hussain, M.I. and Ikram, M. (2012) Entanglement Engineering of a Close Bipartite Atomic System in a Dissipative Environment. Journal of Physics B, 45, Article ID: 115503. https://doi.org/10.1088/0953-4075/45/11/115503

[9] Yu, T. and Eberly, J.H. (2006) Sudden Death of Entanglement: Classical Noise Effects. Optics Communications, 264, 393-397. https://doi.org/10.1016/j.optcom.2006.01.061

[10] Yu, T. and Eberly, J.H. (2004) Finite-Time Disentanglement via Spontaneous Emission. Physical Review Letters, 93, Article ID: 140404.

https://doi.org/10.1103/PhysRevLett.93.140404 
[11] Ban, M., Kitajima, S. and Shibata, F. (2005) Masashi Ban and Sachiko Kitajima and Fumiaki Shibata. Journal of Physics A: Mathematical and General, 38, 4235. https://doi.org/10.1088/0305-4470/38/19/012

[12] Milburn, G.J. (1991) Intrinsic Decoherence in Quantum Mechanics. Physical Review $A$, 44, 5401-5406. https://doi.org/10.1103/PhysRevA.44.5401

[13] Klionsky, D.J., Abdelmohsen, K., Zorzano, A. and Zughaier, S.M. (1991) Guidelines for the Use and Interpretation of Assays for Monitoring Autophagy. Autophagy, 12, 1-222. https://doi.org/10.1080/15548627.2015.1100356

[14] Jurez Amaro, R., Escudero Jimnez, J.L. and Moya Cessa, H. (2009) Intrinsic Decoherence in the Interaction of Two Fields with a Twolevel Atom. Annalen der Physik, 18, 454-458. https://doi.org/10.1002/andp.200810356

[15] Guo, J. and Song, H. (2008) Effects of Inhomogeneous Magnetic Field on Entanglement and Teleportation in a Two-Qubit Heisenberg XXZ Chain with Intrinsic Decoherence. Physica Scripta, 78, Article ID: 045002.

https://stacks.iop.org/1402-4896/78/i=4/a=045002 https://doi.org/10.1088/0031-8949/78/04/045002

[16] Zidan, N. (2013) Quantum Teleportation via Two-Qubit Heisenberg XYZ Chain. Canadian Journal of Physics, 92, 406-410. https://doi.org/10.1139/cjp-2013-0404

[17] Zhou, L., Song, H.S., Guo, Y.Q. and Li, C. (2003) Enhanced Thermal Entanglement in an Anisotropic Heisenberg XYZ Chain. Physical Review A, 68, Article ID: 024301. https://doi.org/10.1103/PhysRevA.68.024301

[18] Arnesen, M.C., Bose, S. and Vedral, V. (2001) Enhanced Thermal Entanglement in an Anisotropic Heisenberg XYZ Chain. Physical Review Letters, 87, Article ID: 017901. https://doi.org/10.1103/PhysRevLett.87.017901

[19] Wang, X. (2001) Enhanced Thermal Entanglement in an Anisotropic Heisenberg XYZ Chain. Physical Review A, 64, Article ID: 012313. https://doi.org/10.1103/PhysRevA.64.012313

[20] Werlang, T. and Rigolin, G. (2010) Thermal and Magnetic Quantum Discord in Heisenberg Models. Physical Review A, 81, Article ID: 044101.

https://doi.org/10.1103/PhysRevA.81.044101

[21] Werlang, T., Rigolin, G., Trippe, C. and Ribeiro, G.A.P. (2010) T Quantum Correlations in Spin Chains at Finite Temperatures and Quantum Phase Transitions. Physical Review Letters, 105, Article ID: 095702. https://doi.org/10.1103/PhysRevLett.105.095702

[22] Mi, Y., Zhang, J., Song, H. and Guo, J. (2011) Thermal Quantum Discord of Spins in an Inhomogeneous Magnetic Field. Journal of Physics B: Atomic, Molecular and Optical Physics, 44, Article ID: 065504.

https://stacks.iop.org/0953-4075/44/i=6/a=065504 https://doi.org/10.1088/0953-4075/44/6/065504

[23] Zidan, N. (2016) Dynamics of Correlations in the Presences of Intrinsic Decoherence. International Journal of Theoretical Physics, 55, 1274-1284. https://doi.org/10.1007/s10773-015-2768-y

[24] Luo, S. (2005) Entanglement Properties of a Two-Qubit, Mixed-Spin, Heisenberg Chain under a Nonuniform Magnetic Field. Physical Review A, 72, Article ID: 042110. https://doi.org/10.1103/PhysRevA.72.042110

[25] Song, L. and Yang, G. (2014) Entanglement and Measurement-Induced Disturbance about Two-Qubit Heisenberg XYZ Model. International Journal of Theoretical Physics, 53, 1985-1992. https://doi.org/10.1007/s10773-014-2005-0 